\title{
SIMULATION OF GRAVITY CURRENTS BY FLUX-DIFFERENCE SPLITTING SCHEME
}

\author{
Akhilesh Kumar JHA ${ }^{1}$, Juichiro AKIYAMA ${ }^{2}$ and Masaru URA ${ }^{3}$ \\ ${ }^{1}$ Member of JSCE, Dr. Engr., Instructor, Dept. of Civil Engineering, Kyushu Institute of Technology \\ (Sensui Cho 1-1, Tobata, Kitakyushu 804-8550, Japan) \\ ${ }^{2}$ Member of JSCE, Ph.D., Professor, Dept. of Civil Engineering, Kyushu Institute of Technology \\ ${ }^{3}$ Member of JSCE, Dr. Engr., Professor, Dept. of Civil Engineering, Kyushu Institute of Technology
}

\begin{abstract}
The evolution of gravity currents is simulated by a flux-difference splitting scheme implemented on the shallow water and mass conservation equations with modified acceleration due to gravity. The shallow water and mass conservation equations are solved in a decoupled manner. Available empirical relationships are used to enforce the front condition for correcting celerity of the gravity current front. The motion of the gravity currents, made of saline water or water-particle mixture is computed by the model as well as observed experimentally. The computed front velocity and front height are compared with experimental data. Effects of different front condition on the front velocity and height are also investigated. Conclusions on the applicability and the accuracy of shallow water equations solved by flux-difference splitting scheme in case of gravity currents are drawn.
\end{abstract}

Key Words : buoyant cloud, numerical simulation, LES, cubic spline, Smagorisnky SGS

\section{INTRODUCTION}

Gravity current is the flow of a fluid of higher density, under the influence of gravity, into another fluid of lower density. Examples of gravity currents in nature are the turbidity currents, discharge of industrial waste into rivers and sea etc. Gravity currents similar to these natural phenomena can also be generated in a laboratory by suddenly removing the barrier separating two fluids of different densities. While the lighter fluid may be the fresh water, the denser fluid may be saline water or water-particle mixture. The instances of gravity currents abound in nature and, therefore, the importance of studying various aspects of their motion needs no overemphasis ${ }^{1}$.

The numerical investigation of gravity currents can be treated as an initial-value problem and can be computed by shallow water equations using a suitable numerical scheme. This approach focuses on the evolution of gravity currents from a given state, generally the state of rest. In another approach, the steady-state characteristics of an established gravity current are investigated. This approach, independent of the shallow-water theory, takes the steady state of a gravity current as a fact and derives the corresponding relationships that must hold for such a steady state. In most gravity current problems of concern, such as intrusion of fresh or salt water in the vicinity of locks and estuaries, spreading of oil spills etc., the initial state is well defined and the investigation can focus on the evolution of the flow when two fluids of different densities come into contact.

The so-called dam-break problem in shallow water theory can be seen as a gravity current problem in water-air system, wherein the density difference is very large. With this understanding, a similar set of governing equations may be applied to the systems with much lesser density difference using a suitably reduced value for the acceleration due to gravity. This approach has been adopted by previous researchers ${ }^{2,3)}$ but the results presented in these were not verified against experimental data.

This paper simulates the evolution of gravity currents by the shallow water equations with modified acceleration due to gravity. The flux-difference splitting technique, which has been found very accurate in case of dam-break problems with water-air system, is used in the finite difference scheme for solving the governing equations. The flux-difference splitting technique is also applied to 
the mass conservation equation, which is solved independently of the shallow water equations. The available empirical relationships are investigated for their applicability as the front condition ${ }^{4,5)}$. The model is applied to gravity currents made of saline water and water-particle mixture. Computed front velocity and front height are compared with the experimental data and useful conclusions regarding the use of shallow water equation for computing evolution of gravity currents are presented.

\section{MODEL DESCRIPTION}

The reduced acceleration due to gravity for gravity currents, created by the release of well-mixed saline or water-particle mixture with bulk density $\rho_{0}$ into a sufficiently deep ambient fluid of lesser density $\rho_{a}$, can be expressed as,

$$
\mathrm{g}^{\prime}=\left(\frac{\rho_{0}-\rho_{\mathrm{a}}}{\rho_{\mathrm{a}}}\right) \mathrm{g}
$$

where $\mathrm{g}$ is the acceleration due to gravity. The motion of such a gravity current in a channel can be described by the one-dimensional shallow water equation which can be written in vector form as

$$
\frac{\partial \mathbf{U}}{\partial \mathrm{t}}+\frac{\partial \mathbf{E}}{\partial \mathrm{x}}+\mathbf{S}=0
$$

The vectors, for the case of rectangular, horizontal channel, are given as

$$
\begin{gathered}
U=\left(\begin{array}{c}
h \\
u h
\end{array}\right) ; \quad E=\left(\begin{array}{c}
u h \\
u^{2} h+0.5 S_{1} g^{\prime} h^{2}
\end{array}\right) \\
S=\left(\begin{array}{c}
v_{e} \\
-g^{\prime} h S_{f}
\end{array}\right)
\end{gathered}
$$

where $h$ is the flow thickness, $u$ is the velocity, $S_{1}$ is the coefficient related to density profile, $v_{\mathrm{e}}$ is the entrainment velocity and $S_{f}$ is the friction slope. The entrainment velocity, $\mathrm{v}_{\mathrm{e}}$ in Eq.(3) is computed as

$$
\mathrm{v}_{\mathrm{e}}=\mathrm{E}_{\mathrm{d}} \mathrm{u}
$$

where $E_{d}$ is the entrainment coefficient. The entrainment coefficient is computed by ${ }^{6}$

$$
\mathrm{E}_{\mathrm{d}}=\frac{0.08-0.1 \mathrm{R}_{\mathrm{i}}}{1+5 \mathrm{R}_{\mathrm{i}}}
$$

where $R_{i}$ is the Richardson number defined as

$$
\mathrm{R}_{\mathrm{i}}=\frac{\mathrm{g}^{\prime} \mathrm{h}}{\mathrm{u}^{2}}
$$

The flux vector $\mathbf{E}$ is related to flow vector $\mathbf{U}$ through its Jacobian $\mathbf{J}$ as

$$
\mathbf{E}=\mathbf{J} \frac{\partial \mathbf{U}}{\partial \mathbf{x}} ; \quad \mathbf{J}=\left[\begin{array}{cc}
0 & 1 \\
\mathrm{~g}^{\prime} \mathrm{h}-\mathrm{u}^{2} & 2 \mathrm{u}
\end{array}\right]
$$

The governing equations are known to be hyperbolic which means that $\mathbf{J}$ has a complete set of independent and real eigenvectors expressed as

$$
\mathbf{e}^{1,2}=\left(\begin{array}{c}
1 \\
\mathrm{u} \pm \mathrm{c}
\end{array}\right) ; \quad \mathrm{c}=\sqrt{\mathrm{g}^{\prime} \mathrm{h}}
$$

where $\mathrm{c}$ is the celerity. The eigenvalues of $\mathbf{J}$ are given by

$$
\lambda^{1,2}=\mathrm{u} \pm \mathrm{c}
$$

Roe's ${ }^{7)}$ averages for velocity and celerity, as given below, are used to construct an approximate Jacobian for conservative evaluation $\mathbf{E}$ in Eq.(7a)

$$
\begin{gathered}
u_{i \pm 1 / 2}=\frac{h_{i \pm 1}^{1 / 2} u_{i \pm 1}+h_{i}^{1 / 2} u_{i}}{h_{i \pm 1}^{1 / 2}+h_{i}^{1 / 2}} \\
c_{i \pm 1 / 2}^{2}=0.5 g^{\prime}\left(h_{i}+h_{i \pm 1}\right)
\end{gathered}
$$

Eq.(2) is integrated by Roe's flux difference splitting scheme ${ }^{7)}$ written as

$$
\mathbf{U}_{\mathrm{i}}^{\mathrm{t}+1}=\mathbf{U}_{\mathrm{i}}^{\mathrm{t}}-\gamma\left(\mathbf{F}_{\mathrm{i}+1 / 2}^{\mathrm{t}}-\mathbf{F}_{\mathrm{i}-1 / 2}^{\mathrm{t}}\right)
$$

where $\mathrm{i}$ and $\mathrm{t}$ are the space and time indices, respectively, $\gamma=\Delta \mathrm{t} / \Delta \mathrm{x}, \Delta \mathrm{t}$ is the time increment and $\Delta \mathrm{x}$ is the finite difference grid size in space. All variables in the following are computed at known time level t. $\mathbf{F}_{\mathrm{i}+1 / 2}$ and $\mathbf{F}_{\mathrm{i}-1 / 2}$ are called numerical fluxes and are expressed as

$$
\begin{aligned}
\mathbf{F}_{\mathrm{i} \pm 1 / 2} & =0.5\left(\mathbf{E}_{\mathrm{i}}+\mathbf{E}_{\mathrm{i} \pm 1}\right) \\
& -0.5 \sum_{\mathrm{k}=1}^{2}\left|\lambda_{\mathrm{i} \pm 1 / 2}^{\mathrm{k}}\right| \alpha_{\mathrm{i} \pm 1 / 2}^{\mathrm{k}} \mathbf{e}_{\mathrm{i} \pm 1 / 2}^{\mathrm{k}}
\end{aligned}
$$

where $\alpha$ is the wave'strength, defined as

$$
\begin{aligned}
\alpha_{\mathrm{i} \pm 1 / 2}^{1,2} & =\mathbf{e}_{\mathrm{i} \pm 1 / 2}^{-1} \Delta \mathbf{U}_{\mathrm{i} \pm 1 / 2} ; \\
\Delta \mathbf{U}_{\mathrm{i}+1 / 2} & =\mathbf{U}_{\mathrm{i}+1}-\mathbf{U}_{\mathrm{i}}
\end{aligned}
$$

In solving Eq.(12) as such, it has to be noted that the front of the gravity current would move with a celerity $2\left(\mathrm{~g}^{\prime} \mathrm{h}_{0}\right)^{1 / 2}$, where $h_{0}$ is the initial height of the density cloud, similar to a standard dam-break wave on a dry bed. However, it is known that the front of a gravity current moves with a much slower celerity than that. This information 
must be incorporated into the model for a realistic modeling of the gravity current. This is achieved by imposing a suitable condition at the moving front of the gravity current. The front condition, in terms of densimetric Froude number, can be written as

$$
\mathrm{U}_{\mathrm{f}}=\mathrm{F}_{\mathrm{ri}} \sqrt{\mathrm{g}^{\prime} \mathrm{h}_{\mathrm{f}}}
$$

The densimentric Froude number $F_{\mathrm{ri}}$ of the front can be computed by an empirical formula. Among the widely acknowledged empirical formulae for the densimetric Froude number, we have tried the one given by Huppert and Simpson ${ }^{4)}$ and another by Benjamin $^{5)}$. The Huppert and Simpson ${ }^{4)}$ formula is given as

$$
\begin{aligned}
\mathrm{F}_{\mathrm{ri}} & =1.19, \text { if } \mathrm{H}_{\mathrm{r}}<0.075 \\
& =0.5\left(\mathrm{H}_{\mathrm{r}}\right)^{-1 / 3}, \text { Otherwise }
\end{aligned}
$$

where $H_{r}=h_{f} / H, h_{f}$ is the front height and $H$ is the ambient water depth. The Benjamin formula can be written as

$$
\mathrm{F}_{\mathrm{ri}}=\left[\frac{2\left(1-\mathrm{H}_{\mathrm{r}}\right)\left(1-0.5 \mathrm{H}_{\mathrm{r}}\right)}{\left(1+\mathrm{H}_{\mathrm{r}}\right)}\right]^{0.5}
$$

Knowing $h_{f}, \mathrm{U}_{\mathrm{f}}$ can be computed from Eq.(14).

Eq.(2) yields the velocity and depth of the density current. Using these results, the equation of mass conservation is solved like a scalar equation by the flux-difference splitting scheme. Allowing loss of mass through downward settling, the mass conservation equation can be written as

$$
\frac{\partial \phi}{\partial \mathrm{t}}+\mathrm{u} \frac{\partial \phi}{\partial \mathrm{x}}=-\mathrm{v}_{\mathrm{s}} \frac{\phi}{\mathrm{h}}
$$

where $\phi$ is the volume fraction occupied by the particles and $\mathrm{v}_{\mathrm{s}}$ is the settling velocity of particles.

It may be noted that the flux vector must be in conservation form for implementing flux-difference splitting technique. Therefore, Eq.(17) is written as

$$
\frac{\partial \phi}{\partial \mathrm{t}}+\frac{\partial(\mathrm{u} \phi)}{\partial \mathrm{x}}=\phi \frac{\partial \mathrm{u}}{\partial \mathrm{x}}-\mathrm{v}_{\mathrm{s}} \frac{\phi}{\mathrm{h}}
$$

and the numerical flux for Eq.(18) becomes ${ }^{8)}$

$$
\begin{aligned}
\mathbf{F}_{\mathrm{i} \pm 1 / 2}= & 0.5\left(\mathbf{E}_{\mathrm{i}}+\mathbf{E}_{\mathrm{i} \pm 1} \pm \varphi_{\mathrm{i} \pm 1 / 2}\right) \\
& \pm 0.5 \sum_{\mathrm{k}=1}^{2}\left|\lambda_{\mathrm{i} \pm 1 / 2}^{\mathrm{k}}\right| \alpha_{\mathrm{i} \pm 1 / 2}^{\mathrm{k}} \mathbf{e}_{\mathrm{i} \pm 1 / 2}^{\mathrm{k}}
\end{aligned}
$$

where $\varphi=\phi \partial u / \partial \mathrm{x}$ and the right hand side of Eq.(17) is evaluated as a source term.

\section{EXPERIMENTS}

Experiments were conducted in a flume made of plexiglass, $7.5 \mathrm{~m}$ long, $0.1 \mathrm{~m}$ wide and $0.9 \mathrm{~m}$ deep.(Fig.1). The gate separating the ambient fluid from either a water-particle mixture or saline water, initial total buoyancy per unit width $\mathrm{W}_{0}$ and initial square shaped area $\mathrm{A}_{0}$, was removed instantaneously to generate gravity currents. Results for three sets of experiments are presented here - two with saline

Table 1: Experimental Conditions

\begin{tabular}{|l|c|c|c|}
\hline Case & $\varepsilon_{0}$ & $\begin{array}{c}\mathrm{W}_{0} \\
\left(\mathrm{~m}^{3} / \mathrm{s}^{2}\right)\end{array}$ & $\begin{array}{c}\mathrm{A}_{0} \\
\left(\mathrm{~m}^{2}\right)\end{array}$ \\
\hline GS2-1 & 0.009 & 0.007938 & 0.0900 \\
GS2-2 & 0.011 & 0.009702 & 0.0900 \\
GP2-1 & 0.040 & 0.004900 & 0.1296 \\
\hline
\end{tabular}

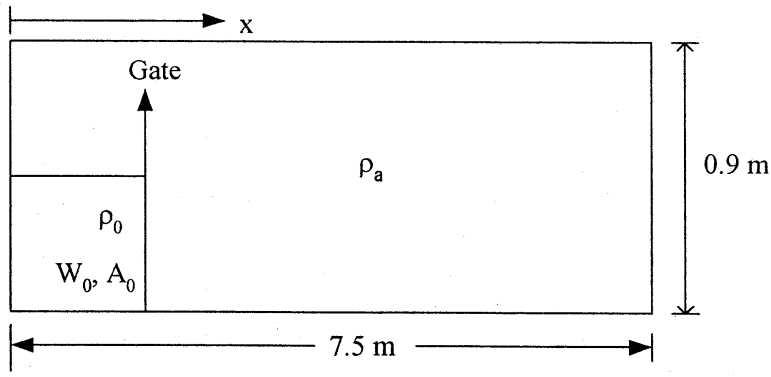

Fig. 1. Experimental setup.
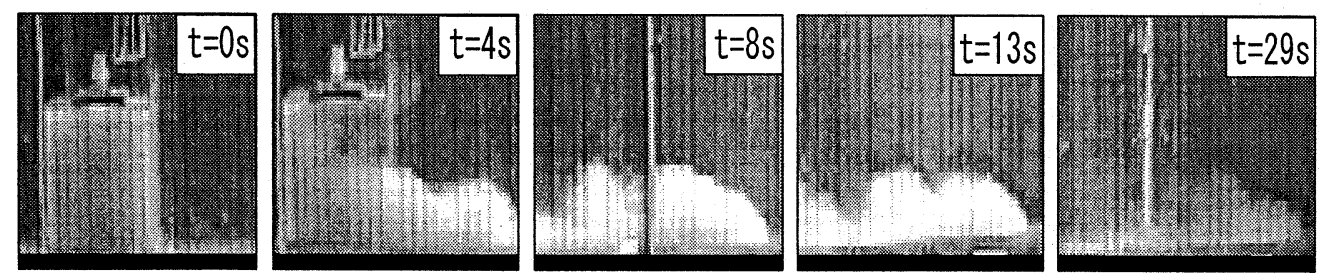

Fig. 2. Evolution of gravity current in the experiment (Case GP2-1). 


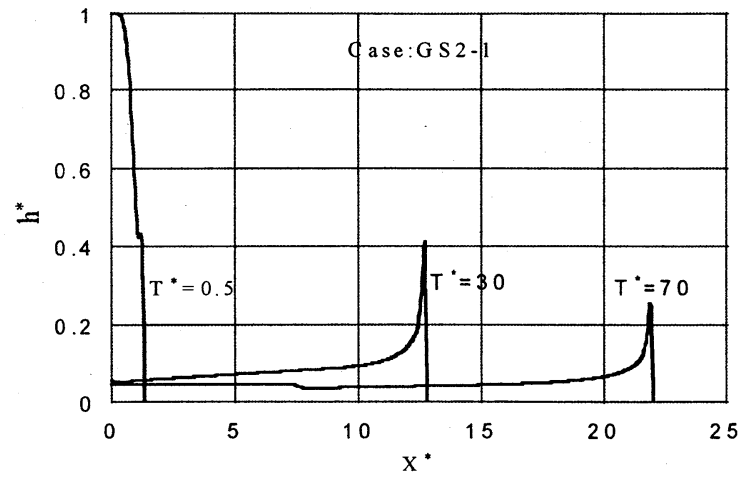

Fig.3 Computed profiles of height of the gravity current.

water and one with water-particle mixture. The depth of ambient fluid $\mathrm{H}$ in all cases was $0.9 \mathrm{~m}$.

Table 1 gives the values of initial buoyancy per unit width $W_{0}$, initial area of the cloud $A_{0}$ and the relative density difference, $\varepsilon_{0}=\left(\rho_{\tilde{0}}-\rho_{\mathrm{a}}\right) / \rho_{\mathrm{a}}$. The notations GS and GP are for experiments with saline water and water-particle mixture, respectively.

The motion of the front of the gravity currents was tracked by a CCD camera and front velocity and front height were obtained from the recorded images. In order to increase the reliability of the data, each experiment was repeated five times under the same experimental conditions and the data presented herein are the average of such five runs. A view of the experiments is provided in Fig.2.

\section{NUMERICAL RESULTS}

The numerical model was first applied to simulate the gravity currents studied in the experiments described above. The Manning's roughness coefficient was set to 0.01 for computing $\mathrm{S}_{\mathrm{f}}$ whereas $\mathrm{S}_{1}$ was set to 0.6. The Huppert and Simpson $^{4)}$ front condition has been used, if not specified otherwise.

It can be seen from experimental view in Fig.2 that it is rather difficult to exactly define the shape of the front region of the gravity current, although approximate front region can be demarcated visually based on experience. The problem becomes more complicated in case of numerical results by the shallow water equation. The front condition acts as a moving wall which forces the gravity current front to move at a rate slower than that given by the shallow water equations. This results in a profile shown in Fig.3, wherein it is almost impossible to

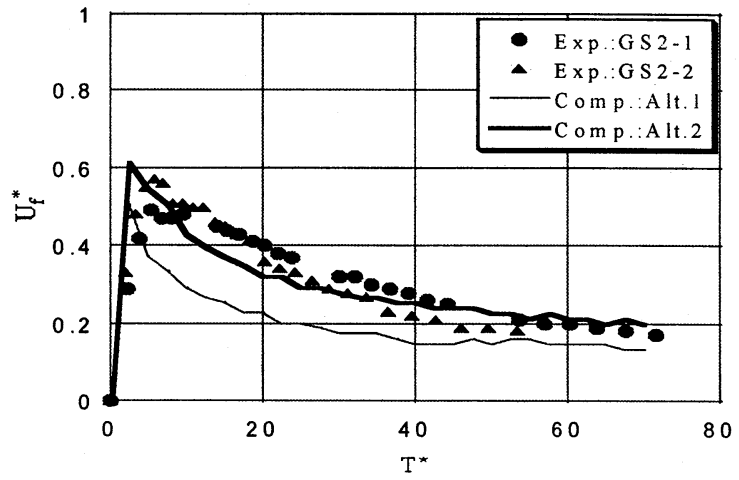

Fig.4 Front velocity for GS2-1 and GS2-2.

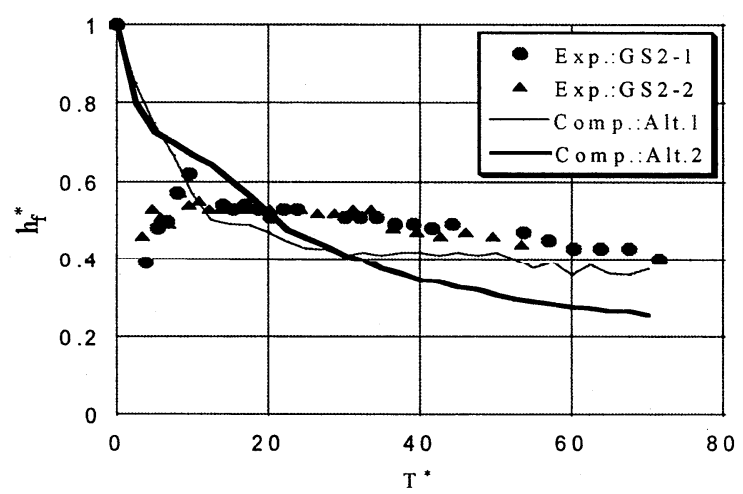

Fig.5 Front height for GS2-1 and GS2-2.

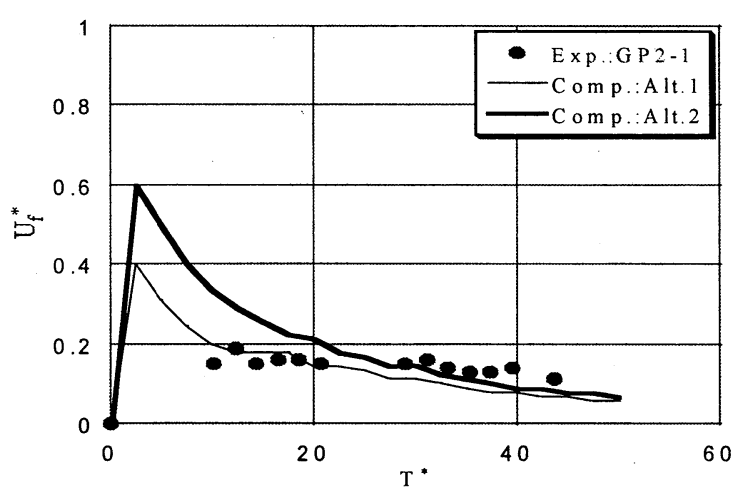

Fig.6 Front velocity for GP2-1.

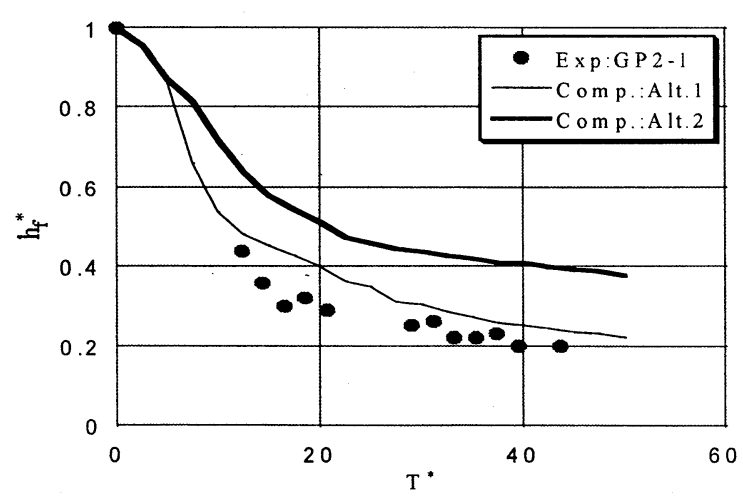

Fig.7 Front height for GP2-1. 
define the front region. The front velocity $U_{f}$ is computed by tracking the leading front while the front height $h_{f}$ is defined as the highest point in the front region. However, the reduced acceleration due to gravity depends on the density of the gravity current, which varies from place to place. The reduced accclcration due to gravity obviously affects both the front velocity and the front height. Generally, the average density of the front region is used to compute reduced acceleration due to gravity. However, in absence of a clear definition of the front region, average front density cannot be computed. Thus, point values for the density of the front must be used. Consequently, the results on front velocity and front height would vary according to the location where reduced acceleration due to gravity is computed.

Two alternatives have been explored in this paper: (1) using the reduced acceleration due to gravity from the location of front height, and (2) using the reduced acceleration due to gravity from a distance one eighths of initial height of the cloud behind the leading point of the gravity current.

The parameters in the results presented herein are non-dimesionalized as: front height and distance by $\left(\mathrm{A}_{0}\right)^{1 / 2}$; front velocity by $\left(\mathrm{W}_{0}^{2} / \mathrm{A}_{0}\right)^{1 / 4}$ and; time by $\left(\mathrm{A}_{0}\right)^{1 / 2} /\left(\mathrm{W}_{0}^{2} / \mathrm{A}_{0}\right)^{1 / 4}$. Non-dimensionalized parameters are indicated by an asterisk in the superscript.

Figs. 4 and 5 compare the experimental and computed front velocities and front heights for Cases GS2-1 and GS2-2. Computed results with Alternative 1 as well as Alternative 2 are shown. It can be seen from the figures that Alternative 1 yields the front heights closer to the experimental data but deviates significantly in case of the front velocities. With Alternative 2, the computed front velocities match well with experimental values but the difference between computed and experimental front heights is larger.

Figs.6 and 7 compare the experimental and computed front velocities and front heights for Case GP2-1. In this case, the particle mass is gradually lost through the downward deposition of the particles. It is observed that the choice of Alternatives 1 or 2 show less pronounced effect on the front velocities but the Alternative 1 gives the front heights closer to the experimental results. The reason or physical meaning of varying performance of Alternative 1 and 2 in case of saline water and water-particle mixture is not clear at this stage.

Next, the effect of the front condition on the front height and the front velocity is examined. Figs. 8 and 9 compare the front velocity and front height by the Huppert and Simpson ${ }^{4)}$ and Benjamin ${ }^{5)}$ front condition for the case of GP2-1. These two are the most commonly used front conditions ${ }^{2,3)}$. The reduced acceleration due to gravity has been computed by Alternative 2. It is observed that the Benjamin front condition yields slightly lower front heights and higher front velocities in the beginning. However, the overall pattern remains more or less

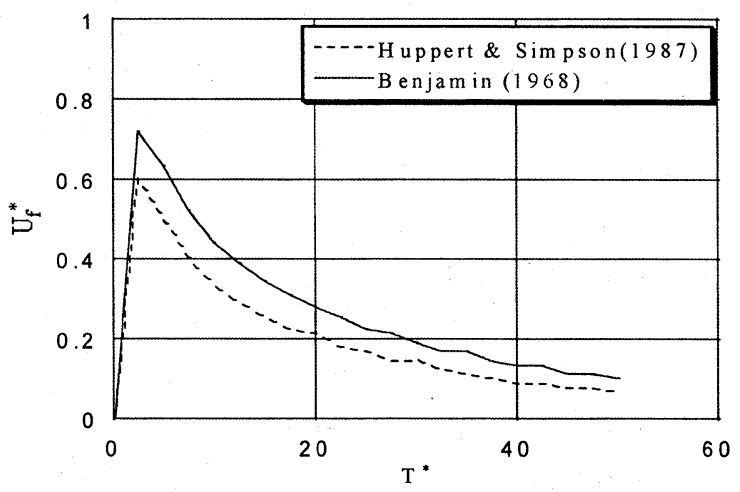

Fig.8 Computed front velocity for GP2-1.

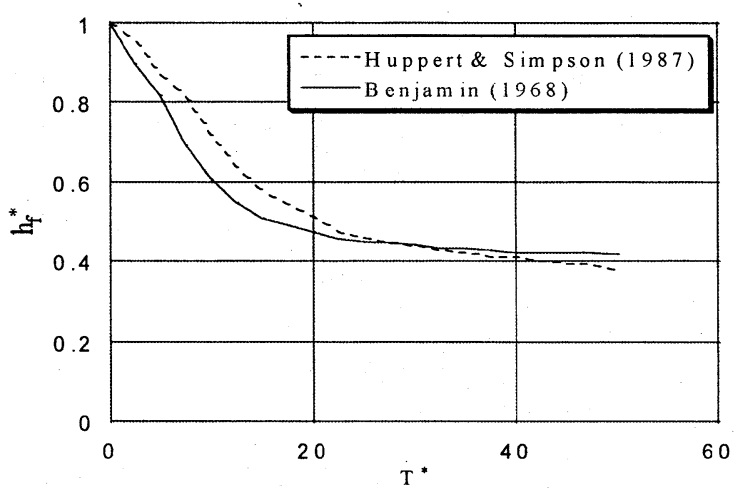

Fig.9 Computed front height for GP2-1.

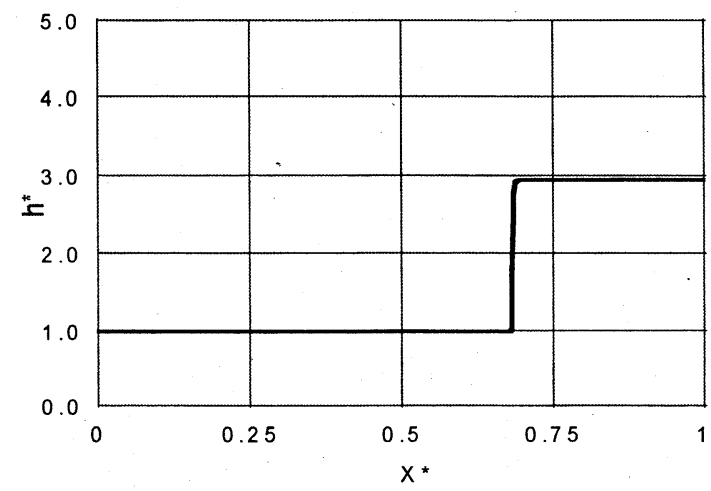

Fig.10 Computed reflected gravity current. 
the same with these two front conditions.

Finally, computational result for the reflection of the gravity current made of salinc watcr from a downstream wall, representing a dam at the downstream end of a reservoir, is presented in Fig.10. The gravity current, defined by $\varepsilon_{0}=0.009$, $\mathrm{W}_{0}=0.007938 \mathrm{~m}^{3} / \mathrm{s}^{2}$ and depth $0.3 \mathrm{~m}$, enters continuously from the upstream end at a steady rate, hits the downstream dam at time zero and gets reflected. A surge is formed which travels upstream leaving still dense mixture behind. The results show the computed profile at $0.5 \mathrm{~s}$ after it hit the wall. Although experimental data is not available to verify quantitatively the accuracy of the computed result, Fig.10 demonstrates the capability of the model for simulating such cases as may be useful to evaluate the extent of the impact of a gravity current running down a reservoir.

\section{CONCLUSIONS}

The evolution of gravity currents has been simulated by solving the shallow water and mass conservation equation with modified acceleration due to gravity using a flux-difference splitting scheme. The densimetric Found number at the front of the gravity current proposed by Huppert and Simpson ${ }^{4)}$ and Benjamin ${ }^{5)}$ have been examined for their use as the front condition, which modifies the front velocity from that in case of the shallow water equations. The numerical model has been applied to simulate the motion of the gravity currents, made of saline water as well as water-particle mixture, and the computed results have been compared with experimental data.

The applicability as well as the limitations of computing evolution of gravity current by shallow water equations have been demonstrated by comparing computed front. velocities and front heights with experimental data. It is concluded, in principle, that the shallow water theory can yield reasonable results on gravity currents with simpler and faster computations compared to the Large-Eddy simulations or the Direct Numerical Simulations. However, the need of an all-important additional front condition considerably limits the scope of this approach. It is also noted that since a well-defined front is not obtained in the computation, it becomes rather arbitrary to define the reduced acceleration due to gravity of the front, which in turn affects the use of an empirical front condition. Therefore, depending on the choice of an cmpirical formula for the front condition and the definition of the average density of the front region, the results might change considerably.

It is also understood that if the interest lies in the continuous flow of the dense fluid in a less denser fluid, wherein the characteristics of front are not of concern, the proposed method may be of better use. The computational results on such flows need to be verified against experimental data.

It is concluded that further work and better understanding of the aforementioned points would greatly enhance the applicability and accuracy of results on gravity currents by shallow water theory.

\section{ACKNOWLEDGMENT}

This study was supported by the Grant-in-Aid for Science Research of the Ministry of Education and Culture, Japan under the Grant (B)12555149 and (C)13650571.

\section{REFERENCES}

1. Simpson, J. E.: Gravity Currents: In the environment and the laboratory, Ellis Horwood, 1987.

2. Bonnecaze, R. T., Huppert, H. E. and Lister, J. R.: Particle-driven gravity currents, J. Fluid Mech., Vol. 250, pp.339-369, 1993.

3. Rottman, J. W. and Simpson, J. E.: Gravity currents produced by instantaneous release of a heavy fluid in a rectangular channel, J. Fluid Mech., Vol. 135, 1983.

4. Huppert, H. E. and Simpson, J. E.: The slumping of gravity currents, J. Fluid Mech. Vol.9, No.4, pp.785-799, 1980.

5. Benjamin, T. B.: Gravity currents and related phenomena, $J$. Fluid Mech., Vol.31, pp.431-471, 1968.

6. Turner, J. S.: Turbulent entrainment: The development of the entrainment assumption, and its application to geophysical flows, J. Fluid Mech., Vol.173, pp.431-471, 1986.

7. Roe, P. L.: Approximate Riemann solvers, parameter vectors and difference schemes, J. Comp. Physics, Vol.43, pp.357-372, 1981.

8. Garcia-Navarro, P. and Vazquez-Cendon, E.: Some considerations and improvements on the performance of Roe's scheme for 1D irregular geometries, Technical Report 23, Dept. de Matematica Aplicada, Universidad de Santiago de Compostela, 1997.

(Received October 1, 2001) 